\title{
Plant peroxisomes: Recent discoveries in functional complexity, organelle homeostasis, and morphological dynamics
}

\section{Sigrun Reumann ${ }^{\mathrm{a}, \mathrm{b}}$ and Bonnie Bartel ${ }^{\mathrm{c}}$}

\author{
${ }^{a}$ Department of Plant Biochemistry and Infection Biology, Biocentre Klein Flottbek, University of \\ Hamburg, D-22609 Hamburg, Germany, sigrun.reumann@uni-hamburg.de \\ ${ }^{\mathrm{b}}$ Centre for Organelle Research, Faculty of Science and Technology, University of Stavanger, \\ $\mathrm{N}-4036$, Stavanger, Norway \\ ${ }^{c}$ Department of BioSciences, Rice University, Houston, Texas 77005, USA, bartel@rice.edu
}

\begin{abstract}
Abbreviations
AGT1, serine:glyoxylate aminotransferase; ATG, AUTOPHAGY-RELATED; ABC, ATP-binding cassette; CAT, catalase; CGI-58, COMPARATIVE GENE IDENTIFICATION-58; DHNA, 1,4dihydroxy-2-naphthoate; ER, endoplasmic reticulum; JA, jasmonate; OPPP, oxidative pentose phosphate pathway; PEX, peroxin; pICDH, peroxisomal NADP-dependent isocitrate dehydrogenase; PMP, peroxisomal membrane protein; PTS, peroxisome-targeting signal; PXA, PEROXISOMAL ABC-TRANSPORTER; RabGAP22, Rab GTPase-activating protein 22; ROS, reactive oxygen species; SDP, SUGAR DEPENDENT.
\end{abstract}




\section{Abstract}

Peroxisomes are essential for life in plants. These organelles house a variety of metabolic processes that generate and inactivate reactive oxygen species. Our knowledge of pathways and mechanisms that depend on peroxisomes and their constituent enzymes continues to grow, and in this review we highlight recent advances in understanding the identity and biological functions of peroxisomal enzymes and metabolic processes. We also review how peroxisomal matrix and membrane proteins enter the organelle from their sites of synthesis. Peroxisome homeostasis is regulated by specific degradation mechanisms, and we discuss the contributions of specialized autophagy and a peroxisomal protease to the degradation of entire peroxisomes and peroxisomal enzymes that are damaged or superfluous. Finally, we review how peroxisomes can flexibly change their morphology to facilitate inter-organellar contacts.

\section{Introduction}

Peroxisomes are small organelles found in most eukaryotes that are delimited by a single lipid bilayer. Diverse metabolic activities are compartmentalized in plant peroxisomes (Table 1). Well-established peroxisomal activities include fatty acid $\beta$-oxidation, hormone production, and photorespiration. Peroxisomal pathways often include an oxidative step generating reactive oxygen species (ROS) as byproducts; peroxisomes therefore also house catalase and other ROS-inactivating enzymes.

Peroxisomes primarily proliferate by growth and division (Figure 1). The proteins required for peroxisome biogenesis, matrix protein import, and division are designated as peroxins (PEX proteins). Peroxisomal matrix proteins are imported into the organelle with the assistance of two interacting receptors, PEX5 for peroxisome-targeting signal type 1 (PTS1) proteins and PEX7 for PTS2 proteins (Figure 2). The cargo-loaded receptors dock at the peroxisomal membrane via interactions with PEX13 and PEX14 and release their cargo into the matrix by largely unknown mechanisms. Membrane-associated PEX5 is ubiquitinated, extracted from the membrane and retrotranslocated back to the cytosol for additional import rounds. After arrival in the matrix, the PTS2 domain is removed, whereas PTS1 proteins retain the signal (Figure 2).

Peroxisomal membrane proteins (PMPs) are inserted into peroxisomes either directly or via the ER membrane, from which pre-peroxisomes can bud and mature by post-translational import of matrix and additional membrane proteins into the organelle (Figure 1). PMPs include a subset of the PEX proteins necessary for import of matrix proteins into the organelle. Mature peroxisomes extend, tubulate, and divide by fission, which is considered to be the predominant mode of peroxisome proliferation (Figure 1).

Although key peroxins and core peroxisomal functions are largely conserved in eukaryotes, there are important differences among fungal, animal, and plant peroxisomes. These 
distinctions underscore the need for a thorough knowledge of peroxisome biology at all levels in the reference plant Arabidopsis thaliana, which is prerequisite for translation to crop plants and microalgae for future agricultural and biotechnological applications. In this article, we highlight recent discoveries that have deepened our understanding of the functional plasticity, biogenesis, degradation, and membrane dynamics of peroxisomes in plants.

\section{Functional diversity of plant peroxisomes}

The metabolic diversity and plasticity of peroxisomes is amazing (Table 1), and unexpected functions of plant peroxisomes continue to be discovered. For instance, peroxisomes house biosynthetic steps of phylloquinone (2-methyl-3-phytyl-1,4-naphtho-quinone or vitamin $\mathrm{K}_{1}$ ), a vital co-factor for electron transfer in photosystem I. Phylloquinone biosynthesis begins in plastids with the synthesis of $o$-succinylbenzoate from chorismate. Peroxisomal enzymes catalyze the next three steps to the double-ring structure of naphthoquinone: i) activation via CoA esterification by acyl-activating enzyme 14, ii) ring cyclization by naphthoate synthase to yield the CoA thioester of 1,4-dihydroxy-2-naphthoate (DHNA) [1], and iii) hydrolysis of DHNACoA by two functionally redundant peroxisomal thioesterases [2]. Phylloquinone biosynthesis is finalized in chloroplasts by DHNA prenylation and methylation. Interestingly, while most phylloquinone biosynthetic enzymes are of cyanobacterial origin, the thioesterases apparently originate from Lactobacillales by horizontal gene transfer [2]. Because none of the three peroxisomal enzymes of phylloquinone biosynthesis produces ROS, it remains to be elucidated why these steps were shifted from chloroplasts to peroxisomes during higher plant evolution.

The first enzyme in biotin synthesis (BioF) is peroxisomal in plants and fungi $[3,4]$, while the subsequent steps are mitochondrial. Moreover, peroxisomal $\beta$-oxidation is required for synthesis of the BioF substrate, pimeloyl-CoA [3,4]. In addition, the enzymes catalyzing the final two steps of mevalonate biosynthesis, 5-phosphomevalonate kinase and mevalonate 5-diphosphate decarboxylase, were recently characterized as peroxisomal in Arabidopsis and Catharanthus roseus [5].

High amounts of photorespiratory $\mathrm{H}_{2} \mathrm{O}_{2}$ are produced during photosynthesis. Both host cells and pathogens can impinge on peroxisomal functions to modulate ROS homoeostasis [6]. For example, Arabidopsis LESION SIMULATING DISEASE1 interacts with catalase via a zinc finger domain and increases peroxisomal catalase activity to negatively regulate programmed cell death [7]. In addition, the Rab GTPase-activating protein RabGAP22 facilitates plant defenses against the soil-borne fungal pathogen Verticillium longisporum. Verticillium infection induces Arabidopsis RabGAP22 expression and triggers RabGAP22 redirection from the nucleus to peroxisomes, where it forms a complex with the photorespiratory enzyme, serine:glyoxylate aminotransferase (AGT1) [8]. The rabgap22-1 mutant displays elevated jasmonate (JA) levels, 
and it will be interesting to learn whether the RabGAP22-AGT1 complex interferes with peroxisomally localized JA biosynthetic enzymes.

Only a few membrane proteins that transport metabolic intermediates and co-factors across the peroxisomal membrane have been identified. One such transporter is PEROXISOMAL ATP binding cassette (ABC) TRANSPORTER 1 (PXA1), which transports various substrates into peroxisomes for $\beta$-oxidation, including fatty acids and lipophilic precursors of the hormones JA and auxin in Arabidopsis [9,10] and barley [11]. Whether transport by PXA1 is regulated and whether the import substrates are CoA esters or free fatty acids was long enigmatic. The $\alpha / \beta$ hydrolase, COMPARATIVE GENE IDENTIFICATION-58 (CGI-58), has emerged as a positive regulator of PXA1 [12]. CGI-58 interacts with PXA1 and promotes PXA1 functions in JA and auxin biosynthesis as well as lipid metabolism in non-seed vegetative tissues but not in germinating seeds [12]. In addition to its transport function, Arabidopsis PXA1 displays intrinsic thioesterase activity that is required for fatty acid transport and metabolism [13], implicating CoA esters rather than free fatty acids as PXA1 substrates. It remains to be resolved at which side of the membrane the CoA ester cleavage occurs and whether CGI-58 activates the transport and/or thioesterase activity of PXA1.

Peroxisomes also function in stomatal opening. The SUGAR-DEPENDENT1 lipase (SDP1) [14] and PXA1 transporter $[9,15]$ are required not only for lipid mobilization during germination but also contribute to stomatal opening [16]. Blue-light-induced stomatal opening is accompanied by reduced oil body volume in Arabidopsis guard cells, and sdp1, pxa1, and cgi58 mutants all display slowed light-induced stomatal opening, presumably because impaired fatty acid catabolism reduces ATP production in these mutants and limits apoplast acidification [16]. These findings provide a rationale for the occurrence of stomatal oil bodies throughout the plant kingdom [16]. Moreover, Arabidopsis mutants deficient in peroxisomal NADP-dependent isocitrate dehydrogenase (pICDH) are compromised in light-induced stomatal opening [17]. pICDH is one of the few peroxisomal matrix sources of NADPH, which is needed for JA biosynthesis and the peroxisomal ascorbate-glutathione cycle. The stomatal defect of picdh is reversible by either an antioxidant such as ascorbate or a nitric oxide scavenger, suggesting that pICDH regulates peroxisomal $\mathrm{H}_{2} \mathrm{O}_{2}$ and/or $\mathrm{NO}$ levels and that peroxisomes are needed in guard cells not only for energy metabolism [16], but also for signaling [17].

In addition to $\mathrm{pICDH}$, the oxidative pentose phosphate pathway (OPPP) is an alternative source of peroxisomal NADPH. The three OPPP enzymes are each encoded by multi-gene families, and the isoforms are located in different compartments. Another layer of subcellular complexity is added for the two peroxisomal isoforms (glucose-6-phosphate dehydrogenase and 6-phosphogluconolactonase), which can be targeted to peroxisomes or plastids depending on thioredoxin and redox balance $[18,19]$. Despite isoform redundancy and the high permeability of 
the peroxisomal membrane for small intermediates, the third peroxisomal OPPP enzyme, 6phosphogluconate dehydrogenase isoform 2 , is required for guided growth of pollen tubes within the style as well as successful pollen tube-ovule interaction and fertilization [20]. This finding provides a possible biochemical rationale for a similar requirement of the docking peroxin PEX13 for fertilization [21]. It will be interesting to learn whether the peroxisomal OPPP is essential for the production of nitric oxide, JA, or an unknown signaling molecule.

Calcium is implicated in regulating peroxisome functions. For example, calcium regulates in vitro dimerization and substrate specificity of the DEG15 protease that removes the N-terminal PTS2 from matrix proteins after entry into the peroxisome [22]. DEG15 dimerization is mediated by the calmodulin-like protein CML3 [23], a peroxisomal protein [24] that contributes to peroxisome metabolism, as evidenced by the slight $\beta$-oxidation defects of a $\mathrm{cm} / 3$ mutant [23]. However, unlike deg15 mutants, which exhibit a complete block in PTS2 processing [25,26], cm/3 mutants process PTS2 proteins like wild type [23], suggesting that the $\beta$-oxidation defects in $\mathrm{cm} / 3$ might stem from additional to-be-discovered roles for CML3 (and presumably calcium) in the peroxisome.

\section{Systematic large-scale plant peroxisome research}

Given the metabolic plasticity and diversity of plant peroxisomes (Table 1), large-scale systematic approaches are needed to thoroughly characterize plant peroxisome functions. Proteome analyses combined with in vivo protein targeting validations are established for plant peroxisomes [27-30] and continue to be expanded, for instance to peroxisomes isolated from etiolated seedlings [31].

Most peroxisomal matrix proteins possess a C-terminal PTS1. Accurately predicting functional PTS1 sequences is challenging because non-canonical targeting signals in higher plants display high variability and few have been experimentally validated. The first plantspecific PTS1 prediction methods uncovered many non-canonical PTS1 examples and corresponding novel matrix proteins [32,33]. More quantitative assessments of plant peroxisomal protein import are beginning, and comparing in silico predictions, semi-quantitative in vivo targeting analyses, and in vitro PEX5 binding affinities of PTS1 variants reveals broad agreement [34]. The first plant-specific web server, PredPlantPTS1 (ppp.gobics.de), for predicting plant PTS1 proteins using these models is available [35]. Due to challenges posed by higher motif and position variability, plant-specific PTS2 prediction algorithms are not yet available.

These large-scale approaches have facilitated the discovery of new peroxisome functions (see above). Systematic phenotypic screening has revealed $\beta$-oxidation-related functions for several newly identified peroxisomal proteins [36,37]. Extending mutant screens to abiotic stress 
conditions combined with co-expression analyses uncovered Arabidopsis peroxisomal proteins involved in drought response, including the LON2 protease (see below) and peroxisomal hydroxypyruvate reductase, which is involved in photorespiration [38].

\section{Peroxisome biogenesis}

A core set of peroxins is conserved in plants, fungi, and mammals. Peroxisomal matrix proteins are imported into the organelle by PEX5 and PEX7, which are interdependent in plants and form a dual receptor complex; PEX5 requires PEX7 for stability [39], and PEX7 requires PEX5 for cargo delivery to the peroxisome [40-42]. Cargo-loaded receptors dock with PEX13 and PEX14 at the peroxisomal membrane (Figure 2), allowing PEX5 to enter the membrane to form a ligand-gated pore that transports cargo into the matrix [43]. Many questions regarding this process remain, including the oligomerization status of the receptors during import, the specific roles of the two docking peroxins, and the mechanism of cargo release. A novel covalent biotin label transfer method applied to in vitro peroxisomal import reveals that an $\mathrm{N}$ terminally truncated version of PEX5, which lacks the PEX7-binding domain but retains PEX14and PTS1-binding regions, initially docks in monomeric form to PEX14 in the peroxisomal membrane [44]. In addition to docking roles, in vitro studies suggest that PEX14-PEX5 interactions facilitate unloading of PTS2 cargo, but not PTS1 cargo, into Arabidopsis peroxisomes [45]. This result implicates the other (non-homologous) docking peroxin, PEX13, in PTS1 cargo release. Intriguingly, PEX13 is an essential gene in Arabidopsis [21] whereas $P E X 14$ null alleles are viable and display some residual matrix protein import [46], indicating that PEX14 facilitates but is not absolutely required for matrix protein import in plants.

After cargo delivery, PEX5 and PEX7 are returned to the cytosol for further import rounds. PEX5 recycling is mediated by monoubiquitination and requires a suite of peroxisomeassociated ubiquitination components (Figure 2): i) the ubiquitin-conjugating enzyme PEX4 tethered to the peroxisome by PEX22 [47], ii) three PMP RING domain ubiquitin-protein ligases (PEX2, PEX10, and PEX12 [48]), and iii) the PEX1-PEX6 heterohexameric ATPase tethered to the peroxisome by PEX26 [reviewed in 49]. Single Arabidopsis mutants defective in several of these peroxins inefficiently remove PEX5 from the membrane after cargo delivery, triggering ubiquitin-dependent PEX5 degradation in pex6 mutants [50,51] or PEX5 accumulation in the membrane in pex4 [51,52] and pex12 mutants [53]. PEX5 levels are restored in pex2 pex6 or pex4 pex6 double mutants [52,54], implicating PEX2 and PEX4 in polyubiquitinating PEX5 for proteasomal degradation when PEX5 retrotranslocation is slowed in Arabidopsis, as in yeast [reviewed in 55]. Little is known about PEX7 recycling in plants, but PEX7 recycling and matrix protein import are impaired when GFP-PEX7 is expressed in Arabidopsis [56]. These defects stem from excessive degradation of unmodified PEX7 and can be prevented by mutation of the 
RabE1c GTPase [56]. It will be interesting to learn whether RabE1c plays a role in receptor recycling in wild-type plants.

PEX5 retention in the peroxisomal membrane appears to impair peroxisome function. For example, decreasing PEX5 degradation in pex6 mutants by mutating PEX4 heightens physiological and molecular defects [52]. Conversely, increasing PEX5 degradation by elevating the growth temperature ameliorates pex4 defects [51]. The ATP-dependence of in vitro PEX5PEX14 docking [44] implies that ATP-driven release of endogenous PEX5 from the peroxisomal membrane is necessary for the docking complex to recruit new cargo-loaded PEX5 moieties, suggesting that docking complex impedance might underlie the detrimental effects of excessive membrane-associated PEX5. However, slightly reducing PEX13 expression, which is expected to reduce PEX5 docking, ameliorates pex4 and pex6 physiological defects without notably improving matrix protein import [52], implying that excessive PEX5 retention in the membrane directly impairs peroxisome metabolism, perhaps by allowing leakage of some peroxisome contents.

How membrane proteins reach peroxisomes is less well understood. In Saccharomyces cerevisiae, PEX3 and PEX19 are needed for formation of pre-peroxisomes from the ER and PMP insertion. PEX19 is the receptor that delivers PMPs to PEX3 for insertion. In mammals, PEX16, which is conserved in plants, is needed to recruit PEX3 to the ER [reviewed in 57]. Overexpression of PEX16 derivatives modified to slow ER-to-peroxisome trafficking demonstrates that Arabidopsis PEX16 can recruit PEX3 to the ER in plant cells, as it does in mammals [58]. Although RNAi knockdown of PEX3, PEX16, or PEX19 in Arabidopsis alters peroxisome morphology [59], genetic dissection of early steps in peroxisome biogenesis in plants has been slow because, unlike most PEX genes, PEX3 and PEX19 are duplicated in Arabidopsis, and mutants in these peroxins have not emerged from forward genetic screens. However, disrupting both PEX19 isoforms results in embryonic lethality [60], and PEX16 disruption confers embryonic defects [61] including loss of peroxisomes [62]. Future combinations of reporters that allow PMP visualization in mutants defective in various peroxins is expected to enhance understanding of PMP trafficking.

\section{Matrix protein degradation and pexophagy}

As peroxisomes compartmentalize oxidative reactions, peroxisomal proteins suffer oxidative damage and require turnover (Figure 1). The metabolic transition in germinating seedlings from the glyoxylate cycle to photorespiration is accompanied by the degradation of glyoxylate cycle enzymes $[47,54,63,64]$. This degradation is slowed when fatty acid $\beta$-oxidation is impaired $[63,64]$ and accelerated when catalase is disrupted [64], suggesting that peroxisomal $\mathrm{H}_{2} \mathrm{O}_{2}$ promotes matrix protein degradation. 
Beyond individual matrix proteins, peroxisomes themselves can be degraded via pexophagy, a specialized form of autophagy [reviewed in 65]. In autophagy, cellular constituents are packaged into autophagosomes and sent to the vacuole for degradation. Because autophagy is not essential for life in Arabidopsis [reviewed in 66], peroxisomes can be examined in null mutants incapable of autophagy, including pexophagy. Autophagy-deficient seedlings accumulate peroxisomes under normal growth conditions $[67,68]$. Similarly, tobacco cell cultures degrade peroxisomes via pexophagy not only in carbon-starved but also in optimal growth conditions [69], reflecting pexophagy activity in non-stressed cells. However, glyoxylate cycle enzymes are only slightly stabilized in autophagy mutants [67,70], suggesting that additional pathways exist to degrade these enzymes.

Relationships between pexophagy and matrix protein turnover have been illuminated through studies of the peroxisomal LON2 protease. LON proteins are ATP-dependent homooligomeric proteases [reviewed in 71] that are cytosolic in prokaryotes and organellar in eukaryotes. Mutations in the Arabidopsis peroxisomal LON isoform (LON2) confer peroxisomerelated defects $[26,63]$ that stem from excessive pexophagy; preventing autophagy restores peroxisome numbers and function to lon2 mutants [70,72]. Interestingly, glyoxylate cycle enzymes are destabilized in lon2 mutants but are dramatically stabilized when LON2 and autophagy both are dysfunctional $[70,72]$. This synergy suggests complementary degradation mechanisms; these matrix proteins appear to be degraded by LON2 when autophagy is prevented and by pexophagy when LON2 is disabled. Intriguingly, a protease-dead LON2 variant can rescue the sparse peroxisome phenotype of lon2 mutants but fails to efficiently degrade matrix proteins [72]. In contrast, an ATPase-dead LON2 variant fails to restore normal peroxisome numbers to lon2 but degrades matrix proteins with normal kinetics [72]. These findings suggest that chaperone rather than protease activity of LON2 normally dampens pexophagy.

The recent demonstration of pexophagy in Arabidopsis [67,68,70,73] and tobacco cells [69] prompts the question of which proteins or signaling molecules target peroxisomes for destruction. It will be interesting to identify the LON2 substrates (and perhaps their posttranslational modifications) that trigger pexophagy in lon2 mutants and to learn whether these proteins also promote pexophagy in wild-type plants. Inactive catalase accumulates in peroxisomes of autophagy-defective mutants [68,73], but aggregated catalase is not the pexophagy signal, because pexophagy proceeds apace even in a mutant lacking the predominant seedling catalase isoform [68]. Ubiquitinated PMPs can target peroxisomes for pexophagy in mammalian cells [74], and yeast peroxisomes are directed to pexophagy in mutants (e.g., pex6) that accumulate polyubiquitinated PEX5 [75], but similar plant studies have 
not been reported. It will be interesting to discover how pexophagy is impacted in various Arabidopsis pex mutants.

Intriguingly, Arabidopsis PEX10 and PEX6 interact with AUTOPHAGY-RELATED8 (ATG8) [76], the ubiquitin-like protein that decorates the nascent autophagosome membrane to mediate autophagic cargo engulfment [reviewed in 66]. This interaction suggests that certain peroxins might promote pexophagy in addition to their roles in matrix protein import. Strikingly, a single amino acid change (G93E) in PEX10 adjacent to the PEX10-ATG8 interaction domain [76] confers seedling lethality when homozygous and aberrant peroxisome morphology when heterozygous [77]. PEX10 carrying this G93E mutation still interacts with ATG8 in bimolecular fluorescence complementation experiments; the additional negative charge suggests that PEX10-ATG8 binding would be increased, perhaps causing excessive targeting of peroxisomes to the autophagy machinery [76]. It will be interesting to learn whether disabling autophagy abrogates the lethality of the pex10-G93E mutant.

\section{Peroxisome morphology dynamics}

Plastids, mitochondria, and peroxisomes were traditionally considered as physically separated spherical or rod-shaped organelles. However, these organelles physically interact, and peroxisomes can alter their shape to increase interaction surfaces between collaborating organelles. During photorespiration, recycling phosphoglycolate to phosphoglycerate requires intensive metabolic intermediate exchange between chloroplasts, peroxisomes, and mitochondria along with coordination of photorespiratory enzyme activities. An intimate physical association between these three compartments can be observed by transmission electron microscopy [78]. Peroxisomes adjacent to chloroplasts change from spherical to elliptical during photorespiration in Arabidopsis leaf mesophyll cells, increasing their interaction area and adhesion, determined by femtosecond laser technology, to the chloroplast surface [79]. This inter-organellar adhesion may provide contact sites that ensure efficient flow of photorespiratory metabolites between organelles. Interestingly, mutations in the RING domain of PEX10, but not other RING peroxins, can disrupt peroxisome-chloroplast interactions and confer photorespiration defects [77,80], again suggesting roles for PEX10 beyond PEX5 recycling.

Plant peroxisomes also form dynamic thin protrusions referred to as peroxules (Figure 1) that extend in response to $\mathrm{H}_{2} \mathrm{O}_{2}$ and hydroxyl radical stress and retract upon stress mitigation [81]. High light intensity induces ROS and increases peroxule-mitochondria interactions [82]. The tubulated peroxisomes become constricted and eventually undergo fission to increase peroxisome numbers [82]. Similarly, cadmium treatment induces cytosolic ROS and rapid PEX11a-dependent peroxule formation, followed by peroxisome elongation and division [83]. Beyond increasing surface and contact areas, peroxule-mediated inter-organellar contacts 
might assist in delivering enzymes from peroxisomes to their final subcellular destination. For example, the major triacylglycerol lipase involved in lipid reserve mobilization during seedling establishment in Arabidopsis, SDP1 (see above) [14], localizes to the peroxisomal membrane in young seedlings before traveling to its site of action on the oil body surface. This relocalization is accompanied by peroxule extensions from peroxisomes to oil bodies and facilitated by the core retromer complex [84], which is best known for roles in endosomal protein trafficking [reviewed in 85]. It will be interesting to learn if additional instances of peroxule dynamics involve inter-organellar protein trafficking or the retromer complex.

\section{Conclusions and future directions}

Although combining genetics and biochemistry with large-scale bioinformatics and proteomics has vastly improved our knowledge of plant peroxisome functions and dynamics, much remains to be discovered. The array of physiological functions of plant peroxisomes is amazingly wide compared to fungi and animals. Functions are unknown for several newly identified matrix proteins, and functional mechanisms need to be assembled from fragmentary information, such as the role of plant peroxisomes in biotic and abiotic stress responses. Several peroxisomal metabolite transporters remain to be identified, and deciphering the posttranslational regulation that plant peroxisomes use to coordinate metabolite flow is only beginning. Our understanding of peroxisome biogenesis from the ER remains rudimentary, as neither pre-peroxisomes nor ER-derived vesicles delivering PEX proteins to mature peroxisomes have been imaged in plants or animals. Which proteins mediate pre-peroxisome budding from the ER and how cells decide between de novo biogenesis and fission of existing peroxisomes remain to be elucidated. It also is not known how peroxisomes obtain membrane lipids for expansion. Even matrix protein import is incompletely understood. The residual matrix protein import observed in pex14 null mutants [46] implies important roles for the PEX13 docking peroxin, which might be explored using viable pex13 partial loss-of-function alleles $[53,86]$. Moreover, some peroxins, such as PEX10, are implicated in processes in addition to matrix protein import $[76,77,80]$ that may be plant-specific. We are only beginning to understand the roles of peroxules in peroxisome metabolism and inter-organellar communication. Now that it is clear that plant peroxisomes can be degraded by pexophagy, it is important to elucidate how sub-populations of "old" or dysfunctional peroxisomes are specifically identified, spatially separated, and targeted to the pexophagy machinery. As additional biological roles for the large collection of peroxisomal enzymes are uncovered, the importance of this fascinating organelle in diverse facets of plant biology becomes increasingly apparent. 


\section{Acknowledgements}

We thank Kim Gonzalez, Yun-Ting Kao, Roxanna Llinas, Mauro Rinaldi, Andrew Woodward, Zachary Wright, and Pierce Young for comments on the manuscript and apologize to those whose work could not be discussed because of length constraints. Peroxisome-related research in the authors' laboratories is supported by The Research Council of Norway (204822/F20 and 226973 to SR), the EU Marie Curie Initial Training Network (ITN) "Peroxisome Formation, Function and Metabolism" (PerFuMe, project number 316723 to SR), the National Institutes of Health (R01GM079177 to BB), the National Science Foundation (MCB-1516966 to BB), and the Robert A. Welch Foundation (C-1309 to BB).

\section{References and recommended reading}

Papers of particular interest, published within the period of review, have been highlighted as:

- of special interest

•• of outstanding interest

1. Kim HU, van Oostende C, Basset GJ, Browse J: The AAE14 gene encodes the Arabidopsis o-succinylbenzoyl-CoA ligase that is essential for phylloquinone synthesis and photosystem-I function. Plant $J$ 2008, 54:272-283.

2. Widhalm JR, Ducluzeau AL, Buller NE, Elowsky CG, Olsen LJ, Basset GJ: Phylloquinone (vitamin K1) biosynthesis in plants: two peroxisomal thioesterases of Lactobacillales origin hydrolyze 1,4-dihydroxy-2-naphthoyl-CoA. Plant J 2012, 71:205-215.

3. Tanabe Y, Maruyama J, Yamaoka S, Yahagi D, Matsuo I, Tsutsumi N, Kitamoto K:

Peroxisomes are involved in biotin biosynthesis in Aspergillus and Arabidopsis. $J$ Biol Chem 2011, 286:30455-30461.

4. Magliano P, Flipphi M, Arpat BA, Delessert S, Poirier Y: Contributions of the peroxisome and beta-oxidation cycle to biotin synthesis in fungi. J Biol Chem 2011, 286:4213342140.

5. Simkin AJ, Guirimand G, Papon N, Courdavault V, Thabet I, Ginis O, Bouzid S, GiglioliGuivarc'h N, Clastre M: Peroxisomal localisation of the final steps of the mevalonic acid pathway in planta. Planta 2011, 234:903-914.

6. Sorhagen K, Laxa M, Peterhansel C, Reumann S: The emerging role of photorespiration and non-photorespiratory peroxisomal metabolism in pathogen defence. Plant Biol (Stuttg) 2013, 15:723-736.

7. Li Y, Chen L, Mu J, Zuo J: LESION SIMULATING DISEASE1 interacts with catalases to regulate hypersensitive cell death in Arabidopsis. Plant Physiol 2013, 163:10591070.

8. Roos J, Bejai S, Oide S, Dixelius C: RabGAP22 is required for defense to the vascular pathogen Verticillium longisporum and contributes to stomata immunity. PLoS One 2014, 9:e88187.

9. Zolman BK, Silva ID, Bartel B: The Arabidopsis pxa1 mutant is defective in an ATPbinding cassette transporter-like protein required for peroxisomal fatty acid betaoxidation. Plant Physiol 2001, 127:1266-1278. 
10. Nyathi Y, De Marcos Lousa C, van Roermund CW, Wanders RJ, Johnson B, Baldwin SA, Theodoulou FL, Baker A: The Arabidopsis peroxisomal ABC transporter, comatose, complements the Saccharomyces cerevisiae pxa1 pxa2delta mutant for metabolism of long-chain fatty acids and exhibits fatty acyl-CoA-stimulated ATPase activity. J Biol Chem 2010, 285:29892-29902.

11. Mendiondo GM, Medhurst A, van Roermund CW, Zhang XB, Devonshire J, Scholefield D, Fernandez J, Axcell B, Ramsay L, Waterham HR, et al.: Barley has two peroxisomal ABC transporters with multiple functions in beta-oxidation. Journal of Experimental Botany 2014, 65:4833-4847.

12. Park S, Gidda SK, James CN, Horn PJ, Khuu N, Seay DC, Keereetaweep J, Chapman KD, Mullen RT, Dyer JM: The alpha/beta hydrolase CGI-58 and peroxisomal transport protein PXA1 coregulate lipid homeostasis and signaling in Arabidopsis. Plant Cell 2013, 25:1726-1739.

13. De Marcos Lousa C, van Roermund CW, Postis VL, Dietrich D, Kerr ID, Wanders RJ, Baldwin SA, Baker A, Theodoulou FL: Intrinsic acyl-CoA thioesterase activity of a peroxisomal ATP binding cassette transporter is required for transport and metabolism of fatty acids. Proc Natl Acad Sci U S A 2013, 110:1279-1284.

14. Eastmond PJ: SUGAR-DEPENDENT1 encodes a patatin domain triacylglycerol lipase that initiates storage oil breakdown in germinating Arabidopsis seeds. Plant Cell 2006, 18:665-675.

15. Footitt S, Slocombe SP, Larner V, Kurup S, Wu Y, Larson T, Graham I, Baker A, Holdsworth M: Control of germination and lipid mobilization by COMATOSE, the Arabidopsis homologue of human ALDP. EMBO J 2002, 21:2912-2922.

16. McLachlan DH, Lan J, Geilfus CM, Dodd AN, Larson T, Baker A, Horak H, Kollist H, He ZS, Graham I, et al.: The breakdown of stored triacylglycerols is required during lightinduced stomatal opening. Current Biology 2016, 26:707-712.

-• This study provides a rationale for the widespread occurence of oil bodies in guard cells by demonstrating that peroxisomal fatty acid beta-oxidation is required for timely lightinduced stomatal opening. Oil body volume decreases as stomates open, and defects in fatty acid mobilization reduce the apoplast acidification that is required for opening, suggesting that peroxisomal metabolism contributes to the ATP required for stomatal opening.

17. Leterrier M, Barroso JB, Valderrama R, Begara-Morales JC, Sanchez-Calvo B, Chaki M, Luque F, Vinegla B, Palma JM, Corpas FJ: Peroxisomal NADP-isocitrate dehydrogenase is required for Arabidopsis stomatal movement. Protoplasma 2016, 253:403-415.

- An article demonstrating that peroxisomal NADPH is necessary for stomatal opening suggests that peroxisomes contribute to signaling required for stomatal movement.

18. Hölscher C, Meyer T, von Schaewen A: Dual-targeting of Arabidopsis 6phosphogluconolactonase 3 (PGL3) to chloroplasts and peroxisomes involves interaction with Trx $\mathbf{m} 2$ in the cytosol. Mol Plant 2014, 7:252-255.

19. Meyer T, Hölscher C, Schwoppe C, von Schaewen A: Alternative targeting of Arabidopsis plastidic glucose-6-phosphate dehydrogenase G6PD1 involves cysteine-dependent interaction with G6PD4 in the cytosol. Plant J 2011, 66:745-758.

20. Hölscher C, Lutterbey MC, Lansing H, Meyer T, Fischer K, von Schaewen A: Defects in peroxisomal 6-phosphogluconate dehydrogenase isoform PGD2 prevent gametophytic interaction in Arabidopsis thaliana. Plant Physiol 2016, 171:192-205.

-• This study revealed an unanticipated role for peroxisomal 6-phosphogluconate dehydrogenase in directional pollen tube growth and fertilization, providing a biochemical explanation for a previous observation that gametophyte interaction is impaired when PEX13 in disabled. 
21. Boisson-Dernier A, Frietsch S, Kim TH, Dizon MB, Schroeder JI: The peroxin loss-offunction mutation abstinence by mutual consent disrupts male-female gametophyte recognition. Curr Biol 2008, 18:63-68.

22. Helm M, Luck C, Prestele J, Hierl G, Huesgen PF, Frohlich T, Arnold GJ, Adamska I, Gorg $A$, Lottspeich $F$, et al.: Dual specificities of the glyoxysomal/peroxisomal processing protease Deg15 in higher plants. Proc Natl Acad Sci U S A 2007, 104:11501-11506.

23. Dolze E, Chigri F, Howing T, Hierl G, Isono E, Vothknecht UC, Gietl C: Calmodulin-like protein AtCML3 mediates dimerization of peroxisomal processing protease AtDEG15 and contributes to normal peroxisome metabolism. Plant Mol Biol 2013, 83:607-624.

24. Chigri F, Flosdorff S, Pilz S, Kolle E, Dolze E, Gietl C, Vothknecht UC: The Arabidopsis calmodulin-like proteins AtCML30 and AtCML3 are targeted to mitochondria and peroxisomes, respectively. Plant Mol Biol 2012, 78:211-222.

25. Schuhmann H, Huesgen PF, Gietl C, Adamska I: The DEG15 serine protease cleaves peroxisomal targeting signal 2-containing proteins in Arabidopsis. Plant Physiol 2008, 148:1847-1856.

26. Lingard MJ, Bartel B: Arabidopsis LON2 is necessary for peroxisomal function and sustained matrix protein import. Plant Physiol 2009, 151:1354-1365.

27. Reumann S, Babujee L, Ma C, Wienkoop S, Siemsen T, Antonicelli GE, Rasche N, Luder F, Weckwerth W, Jahn O: Proteome analysis of Arabidopsis leaf peroxisomes reveals novel targeting peptides, metabolic pathways, and defense mechanisms. Plant Cell 2007, 19:3170-3193.

28. Reumann S, Quan S, Aung K, Yang P, Manandhar-Shrestha K, Holbrook D, Linka N, Switzenberg R, Wilkerson CG, Weber AP, et al.: In-depth proteome analysis of Arabidopsis leaf peroxisomes combined with in vivo subcellular targeting verification indicates novel metabolic and regulatory functions of peroxisomes. Plant Physiol 2009, 150:125-143.

29. Eubel H, Meyer EH, Taylor NL, Bussell JD, O'Toole N, Heazlewood JL, Castleden I, Small ID, Smith SM, Millar AH: Novel proteins, putative membrane transporters, and an integrated metabolic network are revealed by quantitative proteomic analysis of Arabidopsis cell culture peroxisomes. Plant Physiol 2008, 148:1809-1829.

30. Arai Y, Hayashi M, Nishimura M: Proteomic identification and characterization of a novel peroxisomal adenine nucleotide transporter supplying ATP for fatty acid beta-oxidation in soybean and Arabidopsis. Plant Cell 2008, 20:3227-3240.

31. Quan S, Yang P, Cassin-Ross G, Kaur N, Switzenberg R, Aung K, Li J, Hu J: Proteome analysis of peroxisomes from etiolated Arabidopsis seedlings identifies a peroxisomal protease involved in beta-oxidation and development. Plant Physiol 2013, 163:1518-1538.

32. Chowdhary G, Kataya AR, Lingner T, Reumann S: Non-canonical peroxisome targeting signals: identification of novel PTS1 tripeptides and characterization of enhancer elements by computational permutation analysis. BMC Plant Biol 2012, 12:142.

33. Lingner T, Kataya AR, Antonicelli GE, Benichou A, Nilssen K, Chen XY, Siemsen T, Morgenstern B, Meinicke P, Reumann S: Identification of novel plant peroxisomal targeting signals by a combination of machine learning methods and in vivo subcellular targeting analyses. Plant Cell 2011, 23:1556-1572.

34. Skoulding NS, Chowdhary G, Deus MJ, Baker A, Reumann S, Warriner SL: Experimental validation of plant peroxisomal targeting prediction algorithms by systematic comparison of in vivo import efficiency and in vitro PTS1 binding affinity. J Mol Biol 2015, 427:1085-1101. 
- The authors compare in silico predictions to variants of a model targeting sequence to reveal broad agreement between semi-quantitative in vivo targeting analyses and in vitro PEX5 binding affinities.

35. Reumann S, Buchwald D, Lingner T: PredPlantPTS1: A web server for the prediction of plant peroxisomal proteins. Front Plant Sci 2012, 3:194.

36. Cassin-Ross G, Hu J: Systematic phenotypic screen of Arabidopsis peroxisomal mutants identifies proteins involved in beta-oxidation. Plant Physiol 2014, 166:1546-1559.

- A suite of genes predicted via bioinformatics or proteomics to encode novel peroxisomal proteins were interrogated via reverse genetics and a battery of phenotypic assays, which revealed multiple beta-oxidation related functions for seven of the examined genes.

37. Wiszniewski AA, Zhou W, Smith SM, Bussell JD: Identification of two Arabidopsis genes encoding a peroxisomal oxidoreductase-like protein and an acyl-CoA synthetaselike protein that are required for responses to pro-auxins. Plant Mol Biol 2009, 69:503-515.

38. Li J, Hu J: Using co-expression analysis and stress-based screens to uncover Arabidopsis peroxisomal proteins involved in drought response. PLOS One 2015, 10:e0137762.

- The authors document drought-associated transcript level changes for multiple peroxisomal enzymes and find that mutants defective in the LON2 protease or the photorespiratory enzyme hydroxypyruvate reductase 1 display heightened drought susceptibility. These findings implicate photorespiration and peroxisomal quality control in drought survival.

39. Ramón NM, Bartel B: Interdependence of the peroxisome-targeting receptors in Arabidopsis thaliana: PEX7 facilitates PEX5 accumulation and import of PTS1 cargo into peroxisomes. Mol Biol Cell 2010, 21:1263-1271.

40. Hayashi M, Yagi M, Nito K, Kamada T, Nishimura M: Differential contribution of two peroxisomal protein receptors to the maintenance of peroxisomal functions in Arabidopsis. J Biol Chem 2005, 280:14829-14835.

41. Woodward AW, Bartel B: The Arabidopsis peroxisomal targeting signal type 2 receptor PEX7 is necessary for peroxisome function and dependent on PEX5. Mol Biol Cell 2005, 16:573-583.

42. Khan BR, Zolman BK: pex5 mutants that differentially disrupt PTS1 and PTS2 peroxisomal matrix protein import in Arabidopsis. Plant Physiol 2010, 154:16021615.

43. Meinecke M, Cizmowski C, Schliebs W, Kruger V, Beck S, Wagner R, Erdmann R: The peroxisomal importomer constitutes a large and highly dynamic pore. Nat Cell Biol 2010, 12:273-277.

44. Bhogal MS, Lanyon-Hogg T, Johnston KA, Warriner SL, Baker A: Covalent label transfer between peroxisomal importomer components reveals export-driven import interactions. J Biol Chem 2016, $291: 2460-2468$.

-• The authors apply the novel technique of covalent biotin label transfer to in vitro peroxisomal import to reveal that monomeric PEX5 binds first to PEX14 when docking at the peroxisomal membrane.

45. Lanyon-Hogg T, Hooper J, Gunn S, Warriner SL, Baker A: PEX14 binding to Arabidopsis PEX5 has differential effects on PTS1 and PTS2 cargo occupancy of the receptor. FEBS Lett 2014, 588:2223-2229.

- These in vitro studies suggest that PEX14-PEX5 interactions facilitate not only PEX7-PEX5 co-receptor docking to the membrane, but also unloading of PTS2 cargo (but not PTS1 cargo) into Arabidopsis peroxisomes. 
46. Monroe-Augustus M, Ramon NM, Ratzel SE, Lingard MJ, Christensen SE, Murali C, Bartel $\mathrm{B}$ : Matrix proteins are inefficiently imported into Arabidopsis peroxisomes lacking the receptor-docking peroxin PEX14. Plant Mol Biol 2011, 77:1-15.

47. Zolman BK, Monroe-Augustus M, Silva ID, Bartel B: Identification and functional characterization of Arabidopsis PEROXIN4 and the interacting protein PEROXIN22. Plant Cell 2005, 17:3422-3435.

48. Kaur N, Zhao Q, Xie Q, Hu J: Arabidopsis RING peroxins are E3 ubiquitin ligases that interact with two homologous ubiquitin receptor proteins. J Integr Plant Biol 2013, 55:108-120.

49. Hu J, Baker A, Bartel B, Linka N, Mullen RT, Reumann S, Zolman BK: Plant peroxisomes: biogenesis and function. Plant Cell 2012, 24:2279-2303.

50. Zolman BK, Bartel B: An Arabidopsis indole-3-butyric acid-response mutant defective in PEROXIN6, an apparent ATPase implicated in peroxisomal function. Proc Natl Acad Sci U S A 2004, 101:1786-1791.

51. Kao YT, Bartel B: Elevated growth temperature decreases levels of the PEX5 peroxisome-targeting signal receptor and ameliorates defects of Arabidopsis mutants with an impaired PEX4 ubiquitin-conjugating enzyme. BMC Plant Biol 2015, 15:224.

- The authors demonstrate that elevated growth temperature increases proteasomal degradation of PEX5 and reduces the physiological defects of pex4-1 mutants, suggesting that efficient retrotranslocation of PEX5 not only increases PEX5 availability for additional cargo delivery but also prevents peroxisome dysfunction resulting from PEX5 retention in the peroxisomal membrane.

52. Ratzel SE, Lingard MJ, Woodward AW, Bartel B: Reducing PEX13 expression ameliorates physiological defects of late-acting peroxin mutants. Traffic 2011 , 12:121-134.

53. Mano S, Nakamori C, Nito K, Kondo M, Nishimura M: The Arabidopsis pex12 and pex13 mutants are defective in both PTS1- and PTS2-dependent protein transport to peroxisomes. Plant $J$ 2006, 47:604-618.

54. Burkhart SE, Kao YT, Bartel B: Peroxisomal ubiquitin-protein ligases Peroxin2 and Peroxin10 have distinct but synergistic roles in matrix protein import and Peroxin5 retrotranslocation in Arabidopsis. Plant Physiol 2014, 166:1329-1344.

- The authors recover viable Arabidopsis pex2 and pex10 mutants in a screen for peroxisomal matrix protein degradation and implicate PEX2 in PEX5 degradation when retrotranslocation is impaired.

55. Platta HW, Hagen S, Erdmann R: The exportomer: the peroxisomal receptor export machinery. Cell Mol Life Sci 2013, 70:1393-1411.

56. Cui S, Fukao Y, Mano S, Yamada K, Hayashi M, Nishimura M: Proteomic analysis reveals that the Rab GTPase RabE1c is involved in the degradation of the peroxisomal protein receptor PEX7 (peroxin 7). J Biol Chem 2013, 288:6014-6023.

57. Hua R, Kim PK: Multiple paths to peroxisomes: Mechanism of peroxisome maintenance in mammals. Biochim Biophys Acta 2016, 1863:881-891.

58. Hua R, Gidda SK, Aranovich A, Mullen RT, Kim PK: Multiple domains in PEX16 mediate Its trafficking and recruitment of peroxisomal proteins to the ER. Traffic 2015, 16:832-852.

-• This paper demonstrates that variants of human and Arabidopsis PEX16 can recruit multiple PMPs to the ER and delineates the domains in human PEX16 needed for ER targeting, ER-to-peroxisome traficking, and PMP recruitment.

59. Nito K, Kamigaki A, Kondo M, Hayashi M, Nishimura M: Functional classification of Arabidopsis peroxisome biogenesis factors proposed from analyses of knockdown mutants. Plant Cell Physiol 2007, 48:763-774. 
60. McDonnell MM, Burkhart SE, Stoddard JM, Wright ZJ, Strader LC, Bartel B: The earlyacting peroxin PEX19 is redundantly encoded, farnesylated, and essential for viability in Arabidopsis thaliana. PLOS One 2016, 11:e0148335.

61. Lin Y, Sun L, Nguyen LV, Rachubinski RA, Goodman HM: The Pex16p homolog SSE1 and storage organelle formation in Arabidopsis seeds. Science 1999, 284:328-330.

62. Lin Y, Cluette-Brown JE, Goodman HM: The peroxisome deficient Arabidopsis mutant sse1 exhibits impaired fatty acid synthesis. Plant Physiol 2004, 135:814-827.

63. Burkhart SE, Lingard MJ, Bartel B: Genetic dissection of peroxisome-associated matrix protein degradation in Arabidopsis thaliana. Genetics 2013, 193:125-141.

64. Lingard MJ, Monroe-Augustus M, Bartel B: Peroxisome-associated matrix protein degradation in Arabidopsis. Proc Natl Acad Sci U S A 2009, 106:4561-4566.

65. Young PG, Bartel B: Pexophagy and peroxisomal protein turnover in plants. Biochim Biophys Acta 2016, 1863:999-1005.

66. Li F, Vierstra RD: Autophagy: a multifaceted intracellular system for bulk and selective recycling. Trends Plant Sci 2012, 17:526-537.

67. Kim J, Lee H, Lee HN, Kim SH, Shin KD, Chung T: Autophagy-related proteins are required for degradation of peroxisomes in Arabidopsis hypocotyls during seedling growth. Plant Cell 2013, 25:4956-4966.

68. Shibata M, Oikawa K, Yoshimoto K, Kondo M, Mano S, Yamada K, Hayashi M, Sakamoto W, Ohsumi Y, Nishimura M: Highly oxidized peroxisomes are selectively degraded via autophagy in Arabidopsis. Plant Cell 2013, 25:4967-4983.

69. Voitsekhovskaja OV, Schiermeyer A, Reumann S: Plant peroxisomes are degraded by starvation-induced and constitutive autophagy in tobacco BY-2 suspensioncultured cells. Front Plant Sci 2014, 5:629.

70. Farmer LM, Rinaldi MA, Young PG, Danan CH, Burkhart SE, Bartel B: Disrupting autophagy restores peroxisome function to an Arabidopsis lon2 mutant and reveals a role for the LON2 protease in peroxisomal matrix protein degradation. Plant Cell 2013, 25:4085-4100.

71. Gur E: The Lon AAA+ protease. Subcell Biochem 2013, 66:35-51.

72. Goto-Yamada S, Mano S, Nakamori C, Kondo M, Yamawaki R, Kato A, Nishimura M: Chaperone and protease functions of LON protease 2 modulate the peroxisomal transition and degradation with autophagy. Plant Cell Physiol 2014, 55:482-496.

- This paper confirms a previous report that peroxisomes are degraded by autophagy when the LON2 peroxisomal protease is disabled and also assigns different roles to the LON2 protease and ATPase domains in autophagy suppression.

73. Yoshimoto K, Shibata M, Kondo M, Oikawa K, Sato M, Toyooka K, Shirasu K, Nishimura M, Ohsumi Y: Organ-specific quality control of plant peroxisomes is mediated by autophagy. J Cell Sci 2014, 127:1161-1168.

- The authors demonstrate that disfunctional catalase aggregates accumulate in leaf peroxisomes of Arabidopsis mutants incapable of autophagy, suggesting that autophagy normally targets abnormal peroxisomes for destruction.

74. Kim PK, Hailey DW, Mullen RT, Lippincott-Schwartz J: Ubiquitin signals autophagic degradation of cytosolic proteins and peroxisomes. Proc Natl Acad Sci U S A 2008, 105:20567-20574.

75. Nuttall JM, Motley AM, Hettema EH: Deficiency of the exportomer components Pex1, Pex6, and Pex15 causes enhanced pexophagy in Saccharomyces cerevisiae. Autophagy 2014, 10:835-845.

76. Xie Q, Tzfadia O, Levy M, Weithorn E, Peled-Zehavi H, Van Parys T, Van de Peer Y, Galili G: hfAIM: A reliable bioinformatics approach for in silico genome-wide identification of autophagy-associated Atg8-interacting motifs in various organisms. Autophagy 2016, 12:876-887. 
-• This paper uses bioinformatics to find putative ATG8-interacting motifs and applies the technique to identify Arabidopsis peroxins that might interact with ATG8. Several peroxins are predicted to bind ATG8, and the authors use bimolecular fluorescence complementation to confirm ATG8 interactions with PEX6 and PEX10, suggesting that these peroxins might have roles in pexophagy in addition to their established roles in PEX5 recycling.

77. Prestele J, Hierl G, Scherling C, Hetkamp S, Schwechheimer C, Isono E, Weckwerth W, Wanner G, Gietl C: Different functions of the C3HC4 zinc RING finger peroxins PEX10, PEX2, and PEX12 in peroxisome formation and matrix protein import. Proc Natl Acad Sci U S A 2010, 107:14915-14920.

78. Tolbert NE, Essner E: Microbodies: peroxisomes and glyoxysomes. J Cell Biol 1981, 91:271s-283s.

79. Oikawa K, Matsunaga S, Mano S, Kondo M, Yamada K, Hayashi M, Kagawa T, Kadota A, Sakamoto W, Higashi S, et al.: Physical interaction between peroxisomes and chloroplasts elucidated by in situ laser analysis. Nature Plants 2015, 1.

-• The authors apply femtosecond laser technology to quantify interorganellar adhesion. They demonstrate that during photorespiration in Arabidopsis leaf mesophyll cells, peroxisome morphology changes to increase interaction area with adjacent chloroplasts. This increase is accompanied by heightened peroxisome-chloroplast adhesion.

80. Schumann U, Prestele J, O'Geen H, Brueggeman R, Wanner G, Gietl C: Requirement of the C3HC4 zinc RING finger of the Arabidopsis PEX10 for photorespiration and leaf peroxisome contact with chloroplasts. Proc Natl Acad Sci U S A 2007, 104:1069-1074.

81. Sinclair AM, Trobacher CP, Mathur N, Greenwood JS, Mathur J: Peroxule extension over ER-defined paths constitutes a rapid subcellular response to hydroxyl stress. Plant J 2009, 59:231-242.

82. Jaipargas EA, Mathur N, Bou Daher F, Wasteneys GO, Mathur J: High light intensity leads to increased peroxule-mitochondria interactions in plants. Front Cell Dev Biol 2016, 4:6.

- This study shows that high light intensity induces peroxisomal ROS production and reversible extension of peroxules that physically interact with mitochondria.

83. Rodríguez-Serrano M, Romero-Puertas MC, Sanz-Fernández M, Hu J, Sandalio LM: Peroxisomes extend peroxules in a fast response to stress via a reactive oxygen species-mediated induction of peroxin PEX11a. Plant Physiol 2016, 171:1665-1674.

-• The authors show that heavy metal (cadmium or arsenate) treatment triggers rapid PEX11adependent peroxule extension followed by peroxisome proliferation. These changes modulate ROS accumulation, which in turn regulates stress-responsive changes in gene expression.

84. Thazar-Poulot N, Miquel M, Fobis-Loisy I, Gaude T: Peroxisome extensions deliver the Arabidopsis SDP1 lipase to oil bodies. Proc Natl Acad Sci U S A 2015, 112:41584163.

85. Attar N, Cullen PJ: The retromer complex. Adv Enzyme Regul 2010, 50:216-236.

86. Woodward AW, Fleming WA, Burkhart SE, Ratzel SE, Bjornson M, Bartel B: A viable Arabidopsis pex13 missense allele confers severe peroxisomal defects and decreases PEX5 association with peroxisomes. Plant Mol Biol 2014, 86:201-214.

- This paper describes an Arabidopsis pex13 allele that retains sufficient PEX13 function to support viability but displays marked defects in peroxisome-related physiology, matrix protein import, and PEX5 association with peroxisomes. 
Table 1. Plant peroxisome functions.

\begin{tabular}{|c|c|}
\hline Functions & Metabolites or proteins \\
\hline \multicolumn{2}{|l|}{ Catabolic } \\
\hline ROS detoxification & $\mathrm{H}_{2} \mathrm{O}_{2}, \mathrm{O}_{2}^{-\cdot}$ \\
\hline Fatty acid $\beta$-oxidation & Straight-chain, branched, saturated, unsaturated fatty acids \\
\hline Catabolism of primary metabolites & Purines, branched-chain amino acids \\
\hline $\begin{array}{l}\text { Catabolism of secondary } \\
\text { metabolites }\end{array}$ & Polyamines \\
\hline Defense compound metabolism & Indole glucosinolates \\
\hline \multicolumn{2}{|l|}{ Biosynthetic } \\
\hline ROS generation & $\mathrm{H}_{2} \mathrm{O}_{2}, \mathrm{O}_{2}^{--}, \mathrm{NO}$ \\
\hline Glyoxylate cycle & $\mathrm{C}_{4}$ metabolite (succinate) \\
\hline Photorespiration & $\mathrm{C}_{3}$ metabolite and amino acids (glycerate, glycine, serine) \\
\hline Hormone biosynthesis & Jasmonic acid (JA) and indole-3-acetic acid \\
\hline $\begin{array}{l}\text { Co-factor biosynthesis (and } \\
\text { recycling) }\end{array}$ & Phylloquinone, biotin, $\mathrm{NAD}(\mathrm{P}) \mathrm{H}$ \\
\hline Secondary metabolism & Polyamines, benzoic acid, isoprenoids (mevalonate) \\
\hline \multicolumn{2}{|l|}{ Non-metabolic } \\
\hline $\begin{array}{l}\text { Protein processing and } \\
\text { degradation }\end{array}$ & DEG15, LON2 \\
\hline Reversible phosphorylation & CPK1, GPK1, PP2A \\
\hline Calcium signaling & CML3 \\
\hline
\end{tabular}




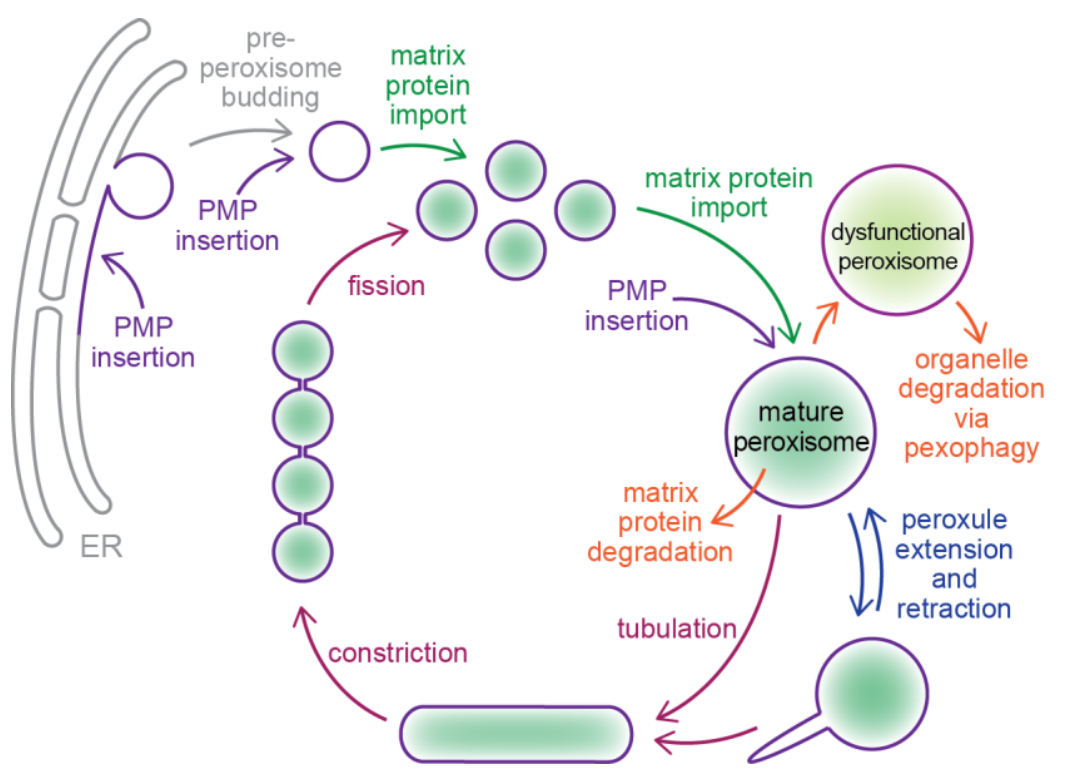

Figure 1. Peroxisome dynamics. Peroxisomal membrane proteins (PMPs) are inserted directly into peroxisomes or into the ER membrane from which pre-peroxisomes can bud. PMPs include a subset of the PEX proteins necessary for import of matrix proteins into the organelle (Figure 2). Mature peroxisomes extend and retract peroxules, tubulate, and divide by fission, which is considered to be the predominant mode of peroxisome proliferation. Peroxisomal quality control includes degrading damaged or obsolete matrix proteins and eliminating dysfunctional or superfluous peroxisomes via pexophagy, a specialized form of autophagy. 


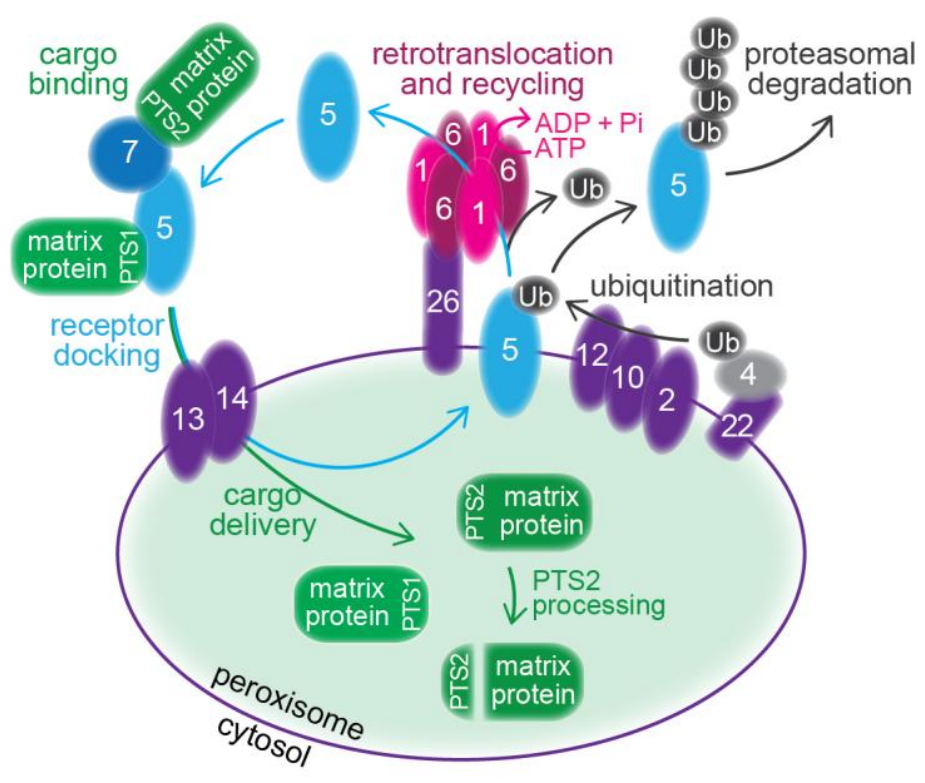

Figure 2. Import of matrix proteins into peroxisomes. Import of peroxisomal matrix proteins (green) is facilitated by peroxins (numbered ovals), some of which are integral membrane proteins (purple). The PEX5 and PEX7 receptors (blue) bind to PTS1 and PTS2 proteins in the cytosol, respectively, and dock at the peroxisomal membrane via interactions with each other and with PEX13 and PEX14. After cargo delivery into the matrix, the N-terminal PTS2 domain is cleaved. Membrane-associated PEX5 is ubiquitinated, presumably with the assistance of the PEX4 ubiquitin (Ub)-conjugating enzyme (gray) and the PEX2-PEX10-PEX12 ubiquitin-protein ligase complex. PEX5 retrotranslocation back to the cytosol for reuse requires the PEX1-PEX6 heterohexameric ATPase (pink). When retrotranslocation is impeded, PEX5 can be polyubiquitinated and degraded by the proteasome. 


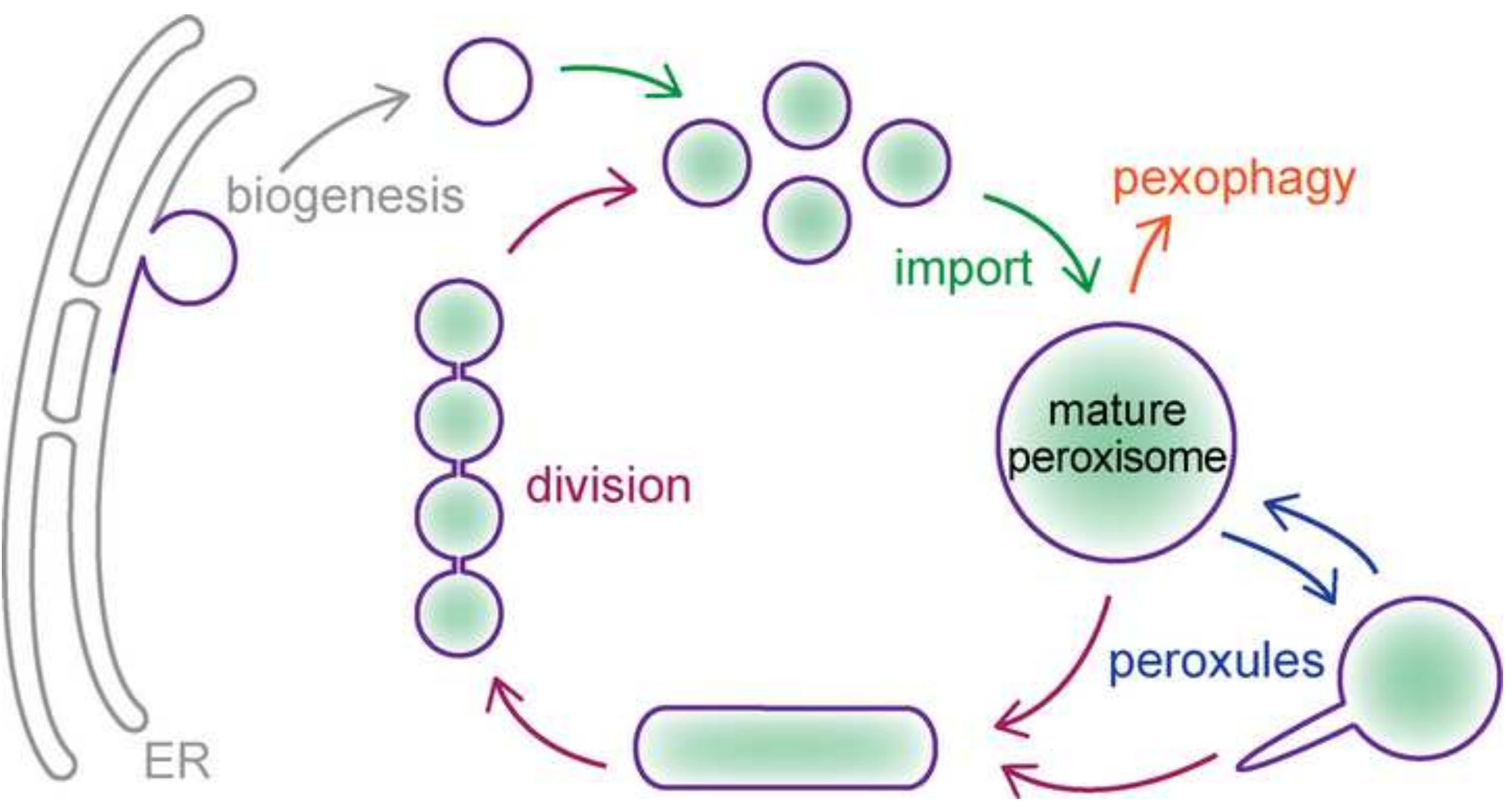

